Enferm Brasil 2021;20(2);222-37

doi: $10.33233 / e b . v 20 i 2.4501$

\title{
REVISÃo
}

\section{Atuação da equipe de enfermagem para o cuidado da população em situação de rua no contexto da pandemia da COVID-19}

Paula Martins Licati*, Lucas Antônio da Silva*, Carolina dos Santos Andrade Diniz*, Danilo de Santana Pereira*, Eduardo Sodré de Souza, D.Sc. **

*Acadêmicos de Enfermagem Universidade Anhembi Morumbi, ${ }^{* *}$ Professor Graduação em Enfermagem Universidade Anhembi Morumbi

Recebido em 5 de dezembro de 2020; Aceito em 5 de março de 2021

Correspondência: Paula Martins Licati, Rua Guiará, 81/46, Pompéia, 05025020 São Paulo SP

\footnotetext{
Paula Martins Licati: paulalicati@gmail.com

Lucas Antônio da Silva: lucas.antonio2013@hotmail.com

Carolina dos Santos Andrade Diniz: caandradesgq@gmail.com

Danilo de Santana Pereira: daniloibitiara@hotmail.com

Eduardo Sodré de Souza: eduardo.souza@anhembi.br
}

\section{Resumo}

Introdução: Dois grandes e importantes eventos do ano de 2020 marcam a história da enfermagem: A comemoração do centenário de Florence Nightingale e o decreto da pandemia da COVID 19. A destacada atuação da enfermagem neste contexto revela sua importância no combate das iniquidades sociais, a exemplo do cuidado à saúde da população em situação de rua. Objetivo: Este estudo tem como objetivo apresentar as ações realizadas pela equipe de enfermagem para o cuidado das pessoas em situação de rua no contexto da pandemia da COVID-19. Métodos: Por meio de revisão integrativa, realizada entre julho e outubro de 2020, na biblioteca virtual em Saúde (BVS). Resultados: Foram identificadas as ações realizadas pelas equipes de enfermagem para minimizar os agravos e propagar os cuidados básicos necessários para reduzir 
a disseminação do vírus entre a população em situação de rua, assim como discutidas as dimensões assistenciais, gerenciais e educativas do processo do trabalho da enfermagem. Conclusão: Foi possível verificar a efetividade da categoria profissional dentro dos serviços de saúde na garantia de desfechos positivos para a saúde da população em situação de rua no contexto da pandemia de COVID-19.

Palavras-chave: equipe de enfermagem; infecção por coronavírus; pandemias; população em situação de rua.

\begin{abstract}
Performance of the nursing team to care for the homeless population in the context of the COVID-19 pandemic

Introduction: Two major and important events in the year 2020 mark the history of nursing: The celebration of the centenary of Florence Nightingale and the pandemic decree of COVID 19. The outstanding performance of nursing in this context reveals its importance in combating social inequities, like the health care of the homeless population. Objective: This study aims to present the actions taken by the nursing team to care for people on the street in the context of the COVID-19 pandemic. Methods: Through an integrative review, carried out between July and October 2020, at the Virtual Health Library (VHL). Results: The actions taken by the nursing teams to minimize the problems and spread the basic care necessary to reduce the spread of the virus among the homeless population were identified. In addition, the care, managerial and educational dimensions of the nursing work process were discussed. Conclusion: It was possible to verify the effectiveness of the professional category within the health services in guaranteeing positive outcomes for the health of the homeless population in the context of the COVID-19 pandemic.
\end{abstract}

Keywords: nursing team; coronavirus infection; pandemic; homeless persons.

\title{
Resumen
}

Desempeño del equipo de enfermería para la atención de la población sin hogar en el contexto de la pandemia COVID-19

Introducción: Dos grandes e importantes hechos del año 2020 marcan la historia de la enfermería: La celebración del centenario de Florence Nightingale y el 
decreto pandémico de COVID-19. El destacado desempeño de la enfermería en este contexto revela su importancia en la lucha contra las inequidades sociales, como la atención médica de la población sin hogar. Objetivo: Este estudio tiene como objetivo presentar las acciones realizadas por el equipo de enfermería para atender a las personas en la calle en el contexto de la pandemia de COVID-19. Métodos: Mediante revisión integradora, realizada entre julio y octubre de 2020, en la Biblioteca Virtual en Salud (BVS). Resultados: Se identificaron las acciones realizadas por los equipos de enfermería para minimizar los problemas y difundir los cuidados básicos necesarios para reducir la propagación del virus entre la población sin hogar, así como se discutieron las dimensiones asistencial, gerencial y educativa del proceso de trabajo de enfermería. Conclusión: Se pudo verificar la efectividad de la categoría profesional dentro de los servicios de salud para garantizar resultados positivos para la salud de la población sin hogar en el contexto de la pandemia COVID-19.

Palabra-clave: grupo de enfermería; infecciones por coronavirus; pandemias; personas sin hogar.

\section{Introdução}

No ano de comemoração do centenário da enfermagem, representado pela Florence Nigthingale, 2020 é um marco para a história da enfermagem. No cenário global atual a enfermagem foi evidenciada como uma categoria profissional essencial para os impactos positivos no que se refere à saúde [1].

Nesse contexto de pandemia da COVID-19, sua atuação marca um histórico de problemas enfrentados diariamente pelos trabalhadores de enfermagem, porém o compromisso ético com o cuidado biopsicossocial ao paciente, família e comunidade permanece [2].

A Organização Mundial de Saúde decretou estado de pandemia da COVID-19 em 11 de março de 2020 e, com o número de pessoas mortas e infectadas, até agora, é possível dizer que assim como outras pandemias, ela também deixará marcas na história da saúde mundial [3].

O Brasil é um dos países com maior número de pessoas mortas e infectadas por COVID-19 do mundo [4]. São Paulo, é o estado que concentra o maior índice da doença [5] cujos efeitos letais são maiores entre populações de 
situação de vulnerabilidade [6], como a população em situação de rua, por exemplo.

Segundo o último censo realizado pela Prefeitura do Estado de São Paulo, a cidade contabilizou cerca de 24.344 mil moradores em situação de rua, maior aumento em vinte anos e 53\% a mais em relação ao ano 2015 que totalizou 15.905 pessoas nesta situação [7].

A Política Nacional para a População em Situação de Rua, através do decreto № 7.053 de 23 de dezembro de 2009, define que a população em situação de rua é [8]:

\footnotetext{
Grupo populacional heterogêneo que possui em comum a pobreza extrema, os vínculos familiares fragilizados ou rompidos e a inexistência de moradia convencional regular. Caracteriza-se pela utilização de logradouros públicos (praças, jardins, canteiros, marquises, viadutos) e de áreas degradadas (prédios abandonados, ruínas, carcaças de veículos) como espaço de moradia e de sustento, de forma temporária ou permanente, bem como das unidades de serviços de acolhimento para pernoite temporário ou moradia provisória [8].
}

A situação de maior vulnerabilidade imposta por esta condição social anuncia a importância do trabalho da enfermagem como uma categoria que atua técnica, ética e cientificamente na produção de um cuidado que visa reduzir as iniquidades em saúde.

Entretanto, o exercício da profissão de enfermagem para a redução das iniquidades, no contexto da pandemia da COVID-19, representa uma fonte de risco que amplia a abrangência dessa vulnerabilidade em saúde. Este cenário lança luz às condições de trabalho destes profissionais, que embora não seja o foco deste trabalho, merece atenção visto que denuncia jornadas de trabalho extensas e inseguras devido a escassez de insumos para o exercício seguro dos procedimentos em saúde [9].

Diante deste cenário de vulnerabilidade em saúde o objetivo deste artigo é apresentar as ações realizadas pela equipe de enfermagem para o cuidado das pessoas em situação de rua no contexto da pandemia da COVID-19. 
Material e métodos

Trata-se de uma revisão integrativa que inclui uma análise ampla de pesquisas publicadas e permite a apreciação de métodos de pesquisa e resultados, permitindo analisar artigos primários e secundários com diferentes tipos de delineamento, focado no tema proposto, sendo necessária uma definição clara e precisa do tema a ser estudado, a fim de tirar conclusões de fácil interpretação [10].

A coleta de dados foi realizada no período de agosto de 2020 a outubro de 2020. Com adaptação da estratégia PICo, uma ferramenta de pesquisa não clínica que utiliza a prática baseada em evidências científicas, foi possível elaborar a pergunta de pesquisa, que auxiliou na pesquisa bibliográfica [11].

A questão norteadora "Quais evidências disponíveis sobre a atuação da equipe de enfermagem para o cuidado da população em situação de rua no contexto da pandemia da COVID-19?" teve como População $(P)=$ equipe de enfermagem; Interesse $(I)=$ população em situação de rua; e Contexto $(\mathrm{Co})=$ pandemia COVID-19.

A busca de artigos na plataforma da Biblioteca Virtual em Saúde (BVS) entre julho e outubro de 2020, contou com os seguintes Descritores em Ciências da Saúde (DECS): "equipe de enfermagem", "cuidados de enfermagem", "enfermagem", "população em situação de rua”, "pandemias", "infecções por coronavírus".

Previamente, foram definidos os seguintes critérios de inclusão: artigos originais completos e gratuitos disponíveis eletronicamente nos idiomas português e inglês. O recorte temporal definido (janeiro a outubro de 2020) considerou o período entre o surgimento dos primeiros casos da infeção no Brasil até o momento da realização das estratégias de buscas de artigos para esta revisão.

Foram recuperados eletronicamente 25 artigos utilizando como estratégia de busca (tw:(cuidados de enfermagem OR enfermagem)) AND (tw:(população em situação de rua)) AND (tw:(pandemias OR infecções por coronavírus)) dos quais 9 foram excluídos após aplicação dos filtros. O restante (11) foi submetido à leitura dos títulos e resumos, sendo excluídos 8 artigos pelos seguintes motivos: tema relacionado ao uso de substâncias e adesão ao tratamento (3); não 
correspondiam com a população em situação de rua (2); e não respondiam à pergunta de pesquisa (3). (Quadro 1).

Quadro 1 - Resultado da busca bibliográfica sobre ações de enfermagem para a saúde da população em situação de rua no contexto de pandemia da COVID19. São Paulo. 2020

\begin{tabular}{|l|l|l|l|l|}
\hline $\begin{array}{l}\text { Base de dados e } \\
\text { Portal }\end{array}$ & $\begin{array}{l}\text { Encontrados } \\
\text { (total) }\end{array}$ & $\begin{array}{l}\text { Total após filtros } \\
\text { e remoção de } \\
\text { duplicidade }\end{array}$ & $\begin{array}{l}\text { Seleção por título } \\
\text { e resumo }\end{array}$ & $\begin{array}{l}\text { Seleção por } \\
\text { análise } \\
\text { completa } \\
\text { (Amostra) }\end{array}$ \\
\hline Portal BVS & 25 & 11 & 8 & 8 \\
\hline MEDLINE & 14 & 6 & 3 & 3 \\
\hline LILACS & 4 & 4 & 4 & 4 \\
\hline Coleciona SUS & 1 & 1 & 1 & 1 \\
\hline Recursos Multimídia & 6 & - & - & - \\
\hline
\end{tabular}

Fonte: Elaborado pelos autores

Foram analisadas, na íntegra, oito publicações, utilizando a análise de conteúdo de Bardin, conforme as seguintes fases: leitura flutuante das informações contidas em cada publicação, codificação, organização das unidades de significado e criação, síntese das informações das publicações [12].

\section{Resultados}

No quadro a seguir são apresentados dados de autoria, ano, periódico, local, tipo do estudo e os principais resultados que correspondem ao objetivo desta revisão (Quadro 2).

Foram identificados quatro artigos na base de dados da Literatura Latinoamericana e do Caribe em Ciências da Saúde (LILACS), três artigos na Medical Literature Analysis and Retrievel System Online (MEDLINE) e um artigo na Coleciona SUS. Estes foram publicados em maior concentração no mês de março (4); entre os meses de maio a agosto foram publicados quatro artigos, um para cada mês correspondente.

Os periódicos com maior número de publicações foram: Coleciona SUS (4), Lancet Respiratory Medicine (1), Instituto de Pesquisa Econômica Aplicada - IPEA (1), Canadian Medical Association Journal - CMAJ (1), e Journal of Urban Health (1). O local com mais publicações foi o Brasil (5) seguida por Inglaterra (1), Canadá (1) e Estados Unidos (1). 
Em relação ao tipo de estudo, todos são de abordagem qualitativa. Em relação aos autores/as destes artigos constam: epidemiologistas (3), médicos/as (5), especialistas em políticas públicas (3) e notas técnicas elaboradas pelo Ministério da Saúde (1) e Secretarias de Saúde (3).

Quadro 2 - Distribuição dos artigos selecionados, autor/ano, revista, local de publicação, tipo de pesquisa e principais resultados. São Paulo, 2020 (ver PDF)

Os principais resultados extraídos foram agrupados conforme as dimensões assistencial, educativa e gerencial do processo de trabalho de enfermagem [21] e serão discutidos na seção a seguir.

\section{Discussão}

A escassez de estudos sobre a enfermagem evidenciada pela ausência de estudos desenvolvidos/autoria de enfermeiras/os e revistas de outras áreas falando sobre a atuação desta categoria profissional demonstra a necessidade de empoderamento desta categoria, uma vez que as equipes de enfermagem são a espinha dorsal de qualquer sistema de saúde [22].

A falta de artigos com dados quantitativos evidencia uma carência de estudos que podem dimensionar a atuação da enfermagem neste contexto, a invisibilidade social desta categoria indica responsabilidade pela desmotivação profissional, devido ao cuidado de longo prazo com o outro, é preciso olhar mais de perto para esses profissionais, é preciso cuidar de quem cuida [23].

Sobre isso, serão discutidas ações de enfermagem que na dimensão assistencial, gerencial e educativa merecem atenção por parte de profissionais e gestores para que o cuidado seja uma forma de produção de saúde que preserve a saúde do cuidador.

Dimensão assistencial do cuidado de enfermagem às pessoas em situação de rua em risco para COVID 19 
Verificou-se que a enfermagem adotou abordagem de atendimento baseada em traumas a fim de persuadir a população de rua a aderirem às orientações de enfermagem [14].

O trauma decorre de eventos que apresentam ameaças existenciais as pessoas, a COVID-19 destaca a ameaça das doenças transmissíveis, uma vez que a população de rua vive diariamente exposta a essas doenças. A enfermagem traz esse tipo de abordagem a fim de persuadi-los tal como acontece com tantos problemas de saúde, com o tratamento irregular e incerto e as vacinas ainda nas fases iniciais de desenvolvimento, lidar com COVID-19 como um vírus diretamente e repleto de complexidade e dificuldade [24].

Ações de enfermagem para identificação de pessoas sintomáticas foram registradas nestes estudos. A triagem em abrigos [15], acolhimento nos serviços de saúde [15], avaliação quanto aos sintomas da COVID-19 [15] e encaminhamento para centros de testagem [15] foram ações articuladas com medidas preventivas e de controle da infecção entre essas pessoas, como: vacinação contra gripe [15]; isolamento da população de rua a fim de evitar surtos [16]; monitoramento de sinais vitais [16]; e identificação precoce de sintomáticos [16].

A importância do acolhimento e classificação de risco, dada a vulnerabilidade da população traz a tona medidas e ações para mitigar os danos a estas pessoas, estratégias de prevenção, intervenção, conscientização e acompanhamento são as medidas mais efetivas analisadas [25].

$O$ trabalho assistencial executado pela enfermagem reforçou a atenção aos sinais e sintomas relacionados à síndrome gripal [17] com extensão dos cuidados para o acompanhamento e monitoramento de assintomáticos e encaminhamento em caso de alterações [17]. A classificação de risco para encaminhamento ao serviço de referência contou com uso de protocolo local de manejo clínico [18].

Os protocolos elaborados para as pessoas em situação de rua durante a pandemia, normatizam ações que as equipes de enfermagem possam atuar [26], mas é muito importante que o enfermeiro tenha flexibilidade no atendimento, ampliando o diálogo social, reconhecendo e respeitando a cultura local, humanizando ações e cuidados, e se necessário realizar manutenção e 
mudanças nas medidas emergenciais para que possam suprir todas as especificidades que esta população demanda [27].

A enfermagem está envolvida nas principais ações de saúde no âmbito da atenção básica, e utiliza da assistência, como parte do trabalho do enfermeiro/a para o desenvolvimento de suas atividades. A aplicação do processo de enfermagem proporciona ao enfermeiro a possibilidade de uma enfermagem humanizada e personalizada [27], cuidando das necessidades básicas da pessoa e respeitando sua cultura, além de ser aplicada na assistência, também pode orientar o enfermeiro na tomada de decisões nas diversas situações vivenciadas pela equipe de enfermagem [28]. Esses fatores estão diretamente alinhados ao direito à saúde garantido a todos os indivíduos a prestação de cuidados da equipe de saúde que garanta universalidade, integralidade e equidade [29].

Durante a assistência de enfermagem o enfermeiro é um dos profissionais que está em contato direto com a população em situação de rua. Dada a complexidade do caso, o conhecimento necessário para prestar o cuidado de enfermagem deve ser adquirido por meio da formação continuada, embora a enfermagem execute procedimentos assistenciais, ela ainda se encontra amarrada a protocolos e diretrizes universalistas, o que se faz necessário uma maior sensibilidade e uma abordagem mais humanizada a questões especificas da população em situação de rua, com isso evita distanciamentos e medos, aprimorando assim a relação da equipe de enfermagem com a população, o que vai requerer uma formação acadêmica mais humanizada, uma maior abordagem na graduação em temas sociais, uma vez que a dimensão educativa também faz parte do processo de enfermagem [30].

\section{Dimensão educativa do cuidado de enfermagem}

Verificou-se que a equipe enfermagem adotou medidas de informação e educação em saúde a fim de manter o distanciamento social e proteger a população de rua [13] e adoção de medidas de cuidado em casos de surgimento e gravidade dos sintomas [17]. Esclarecimentos sobre as formas de contágio, distanciamento social, medidas de higiene, sintomatologia da COVID-19 e fazer 
uso de linguagem coloquial conforme à característica da população [19] foram ações desenvolvidas junto à população em situação de rua.

Educação popular em saúde garante a efetividade das informações, a orientação em saúde é de extrema importância para a prevenção e detecção precoce da doença, reduzindo assim a possibilidade de agravos e riscos de contaminação pela COVID-19, instruir a população de rua destacando a importância do autocuidado é um fator importante para que todos entendam a necessidade da mudança de hábitos [31].

Além de utilizar uma linguagem acessível, a troca entre os saberes científicos, populares e culturais devem acontecer, expondo as informações de forma mais clara e familiar possível, valorizando os saberes dos indivíduos, assim, auxilia na situação e iniciação de tratamentos visando a saúde [32].

Trata-se de um importante recurso que qualifica a assistência de enfermagem, uma vez que a perspectiva dialógica e intersubjetiva na convergência de saberes prevista pela educação popular em saúde permite a aquisição de conhecimentos e o desenvolvimento de técnicas relacionais úteis na execução do cuidado.

\section{Dimensão gerencial do cuidado de enfermagem}

Dentre os equipamentos de saúde, observou-se que a adoção de uma política centralizada no cuidado humanizado, respeitando as particularidades da população em situação de rua, com ações articuladas e seguindo protocolos e normas contribuíra para a redução de danos desses indivíduos [33].

Neste contexto a Política Nacional de Humanização parte dos conceitos e métodos voltados para a reorganização do processo de trabalho em saúde, com foco na transformação das relações sociais, envolvendo trabalhadores e gestores na vivência cotidiana de organização e desenvolvimento dos serviços e mudanças na forma como os serviços são prestados à população [34].

No que tange a equipe de enfermagem, esse contato é construído com respeito, empatia e carinho, focando nas necessidades próprias da população em situação de rua, que vão além de seus meros aspectos fisiopatológicos. 0 enfermeiro precisa estar atento a cultura local, estabelecendo uma sensibilidade cultural, conseguindo assim reconhecer e valorizar as diferenças culturais, 
respeitando valores, crenças e costumes, assim adquirindo conhecimentos necessários e competências tendo em conta as culturas das pessoas, vai ser possível estabelecer cuidados de enfermagem culturalmente competentes que ajudaram a garantir a satisfação desta população, portanto atingirá ganhos em saúde [35].

Aspectos relacionados à garantia de ambientes seguros e adequados para acolhimento e internação de pessoas em situação de rua com risco ou infectados por COVID-19 foram identificados nos estudos. Sobre isso, verificouse a disposição dos leitos com garantia do distanciamento social [14].

Ainda segundo a Política de Humanização ao que se refere a ambiência, a oferta de um espaço físico que garanta aos enfermeiros prestar uma assistência segura, livre de danos e prejuízos faz-se necessária [34].

Em se tratando de segurança do paciente, essa tem uma preocupação prioritária a fim de prevenir danos e reduzir efeitos adversos. A segurança do paciente em situação de rua é um desafio que exige acompanhamento de políticas públicas específicas para refletir sobre ideais, reais cuidados, adaptação e respostas às necessidades dessa população. Os procedimentos de segurança do paciente devem ser traduzidos em metas para aumentar as capacidades das pessoas que vivem nas ruas, e essa adaptação deve incluir comunicação coesa, planejamento e coordenação eficazes, bem como o papel da equipe de enfermagem no cuidado destas pessoas [36].

Em relação à ambiência, à enfermagem coube a provisão de um local específico e seguro para acolhimento, higienização das mãos e manutenção em isolamento social dos casos suspeito ou confirmados [17]. A previsão e provisão de recursos materiais [20] para a higienização dos ambientes [19]; higienização das mãos pelas equipes de profissionais de saúde [19] e higiene e proteção pessoal, para a população em situação de rua [16] foram ações gerenciais da enfermagem identificadas nesta revisão.

Além da garantia de acesso ao serviço a enfermagem na dimensão gerencial, também compete a provisão de materiais o que na pandemia foi dificultado devido à falta de planejamento e financiamento de recursos [37].

Ainda em relação aos aspectos administrativos, verificou-se que 0 trabalho de enfermagem foi organizado de forma que considerou a situação em que estas pessoas vivem, não condicionando o atendimento ou acesso aos 
serviços à apresentação de documentos pessoais como registro geral (RG), Certidão de Pessoa Física (CPF) ou Cartão SUS) [19].

$\mathrm{Na}$ rotina dos serviços, em geral, é comum o acesso aos serviços de saúde estar atrelado a algum documento de identificação pessoal ou cartão SUS, a população em situação de rua sente-se insegura em procurar as unidades públicas de saúde por muitas vezes não portarem tal documentação, o que dificulta a prestação de assistência e adesão de tratamentos para este público [38].

No que se refere a produção do cuidado, a enfermagem tem garantido o acesso da população em situação de rua nos atendimentos de urgência e emergência sem vincular documentos, auxiliando e encaminhando essas pessoas ao serviço social para que seja realizado a continuidade do cuidado, uma vez que a articulação dos serviços se dá mediante cartão SUS, garantindoIhes assim uma maior adesão e redução de danos a essa população [39].

A perspectiva ampla e interprofissional do cuidado, bem como a posição destacada de profissionais de enfermagem na atenção às pessoas em situação de rua, em contexto de pandemia, enfatiza a importância de investimentos em formação acadêmica e em programas de educação permanente que prepare profissionais capazes de atuar de forma autônoma, articulando as dimensões assistencial, gerencial, educativa e investigativa do processo de trabalho da enfermagem.

\section{Conclusão}

Este estudo propôs, como objetivo geral, descrever a atuação da equipe de enfermagem e as ações implementadas durante a pandemia da COVID-19 frente a população em situação de rua.

Observou-se que a equipe de enfermagem é fundamental para fomentar o acesso continuado aos serviços de saúde e prevenir a disseminação da COVID-19 junto a população em situação de rua.

Portanto recomenda-se estudos para aprofundamentos sobre as práticas e teorias de enfermagem que possam resultar em melhores desfechos da saúde da população em situação de rua e agregar mais valor a este campo de conhecimento e prática que por um centenário vem trilhando caminhos plurais 
para a efetivação de um cuidado ético, técnico e estético, compatível com realidades diversas.

\section{Referências}

1. Ribeiro OMPL, Fassarella CS, Trindade LL, et al. Ano internacional da/o enfermeira/o: dos 200 anos de Florence Nightingale à pandemia por Covid-19. Revista de Enfermagem do Centro Oeste Mineiro 2020;10:e3725. doi: 10.19175/recom.v10i0.3725 [Crossref]

2. Quadros A, Fernandes MTC, Araújo BR, Caregnato RCA. Desafios da Enfermagem Brasileira no Combate da COVID-19: uma reflexão. Enfermagem em Foco 2020;11(1). doi: 10.21675/2357-707X.2020.v11.n1.ESP.3748 [Crossref]

3. World Health Organization. Coronavirus disease (COVID-19) outbreak [Internet]. Geneva: World Health Organization; 2020. Available from: https://www.who.int/emergencies/diseases/novel-coronavirus-2019

4. Brasil. Ministério da Saúde. Secretaria de Vigilância em Saúde. Boletim Epidemiológico Especial. COE-COVID19. Available from: https://www.gov.br/saude/ptbr/media/pdf/2020/dezembro/03/boletim epidemiologico covid 39.pdf

5. Coronavírus Brasil. Painel Coronavírus [Internet]. Available from: https://covid.saude.gov.br/

6. Prado MF, Antunes BBP, Bastos LSL. Análise da subnotificação de COVID-19 no Brasil. Rev Bras Ter Intensiva 2020;32(2):224-8. doi: 10.5935/0103-507x.20200030 [Crossref]

7. Prefeitura Municipal de São Paulo. Censo 2029 [internet]. São Paulo; 2019. Available from:

https://www.prefeitura.sp.gov.br/cidade/secretarias/assistencia social/observatorio soci al/pesquisas/index.php? $\mathrm{p}=18626$

8. Brasil. Decreto no 7.053, de 23 de dezembro de 2009. Institui a Política Nacional para a População em Situação de Rua e seu Comitê Intersetorial de Acompanhamento e Monitoramento, e dá outras providências. Brasília, 23 dez 2009b. Available from: http://www.planalto.gov.br/ccivil 03/ ato20072010/2009/decreto/d7053.htm\#: :text=Institui\%20a\%20Pol\%C3\%ADtica\%20Nacional\% 20para,que\%20lhe\%20confere\%200\%20art

9. Fiho JMJ, Assunção AA, Algranti E, Garcia E, Saito CA, Maeno M. A saúde do trabalhador e o enfrentamento da COVID-19. Rev Bras Saúde Ocup 2004; 45:e14. doi: 10.1590/2317-6369ed0000120 [Crossref]

10. Mendes KDS, Silveira RCCP, Galvão CM. Revisão integrativa: método de pesquisa para a incorporação de evidências na saúde e na enfermagem. Texto Contexto Enferm 2008;17(4):758-64. doi: 10.1590/S0104-07072008000400018 [Crossref] 
11. Santos CMDC, Pimenta CADM, Nobre MRC. A estratégia PICO para a construção da pergunta de pesquisa e busca de evidências. Rev Latino-Am. Enfermagem 2007;15(3):508-11. http://doi.org/10.1590/S0104-11692007000300023 [Crossref]

12. Bardin L. Análise de conteúdo Lisboa: Edições 70; 1977.

13. Heimer R, McNeil R, Vlahov D. A Community responds to the COVID-19 Pandemic: a case study in protecting the health and human rights of people who use drugs. J Urban Health 2020;97:448-56. doi: 10.1007/s11524-020-00465-3 [Crossref]

14. Perri M, Dossani H, Hwang SW. COVID-19 and people experiencing homelessness: challenges and mitigation strategies. Canadian Medical Association Journal 2020;192(26):716-19. doi: 10.1503/cmaj.200834 [Crossref]

15. Silva TD, Natalino Pinheiro MB. População em situação de rua em tempos de pandemia: um levantamento de medidas municipais emergenciais Brasília; IPEA; 20200600. 18 p. ilus. (Nota Técnica / IPEA. Disoc, 74). Available from: http://repositorio.ipea.gov.br/bitstream/11058/10078/1/NT 74 Diest Disoc Populacao \%20em\%20Situacao\%20de\%20Rua\%20em\%20Tempos\%20de\%20Pandemia.pdf

16. Kirby T. Efforts escalate to protect homeless people from COVID-19 in UK. The Lancet Respiratory Medicine 2020;8(5):447-9. doi: 10.1016/s2213-2600(20)30160-0 [Crossref]

17. Brasil. Ministério da Saúde. Secretaria de Atenção Primária à Saúde.Prevenção ao Covid-19 no âmbito das equipes de consultórios na rua / Prevention of Covid-19 in the context of street practice teams.Brasília; Brasil. Ministério da Saúde; 2020. Available from: https://www.unasus.gov.br/especial/covid19/pdf/54

18. Santa Catarina (Estado). Secretaria da Saúde. Informações aos profissionais da rede SUS, equipes e gestores no cuidado à saúde das pessoas vivendo em situação de rua para o enfrentamento do Coronavírus / Information to SUS network professionals, teams and managers in health care for people living on the street to cope with the Coronavirus. s.l; s.n; 22 mar. 2020. Available from:

http://docs.bvsalud.org/biblioref/2020/07/1103053/ntc-006-2020-daps-sps-ses-scatualizada-22-03-20.pdf

19. Bahia. Secretaria da Saúde. Superintendência de Vigilância e Proteção da Saúde. Superintendência de Atenção Integral à Saúde. Comitê Estadual de Emergências em Saúde Pública. Orientações para os serviços de saúde na atenção da população em situação de rua do Estado da Bahia voltadas à assistência aos casos suspeitos ou confirmados de infecção pelo novo Coronavírus (SARS-COV-2). Salvador; s.n; 25 mar 2020. 2 p. (Nota Técnica COE Saúde, 25). Available from:

http://docs.bvsalud.org/biblioref/2020/09/1120364/nota tecnica-no25-orientacoespopulacao-de-rua.pdf

20. Rio Grande do Sul (Estado). Secretaria da Saúde. Orientações para o cuidado em saúde à população em situação de rua em razão da disseminação do coronavírus / Guidelines for health care for the homeless population due to the spread of the 
coronavirus.s.I; Rio Grande do Sul (Estado). Secretaria da Saúde; [2020]. Available from: https://www.unasus.gov.br/especial/covid19/pdf/50

21. Kurcgant P. Gerenciamento em enfermagem. Rio de Janeiro: Guanabara Koogan; 2005.

22. Conselho Regional de Enfermagem do Paraná. Demandas de décadas da Enfermagem se sobessaem no enfrentamento à Pandemia da COVID-19. Internet. Paraná: COREN-PR; 2020. Available from: https://www.corenpr.gov.br/portal/noticias/1057-demandas-de-decadas-daenfermagem-se-sobressaem-no-enfrentamento-a-pandemia-da-covid-19

23. Peruzzo S. Conselho Regional de Enfermagem do Paraná. Enfermagem protagonismo invisível socialmente. Internet. Paraná: COREN-PR; 2020. Available from: http://biblioteca.cofen.gov.br/enfermagem-protagonismo-invisivel-socialmente/

24. Johnson MT, Johnson EA, Webber L, Nettle D. Mitigating social and economic sources of trauma: The need for universal basic income during the coronavirus pandemic. Psychol Trauma 2020;2(S1):S191-S92. doi: 10.1037/tra0000739 [Crossref]

25. Honorato BEF, Oliveira ACS. População em situação de rua e COVID-19. Rev Adm Pública 2020;54(4):1064-78. doi: 10.1590/0034-761220200268 [Crossref]

26. Ministério da Cidadania/Secretaria Especial do Desenvolvimento Social/Secretaria Nacional de Assistência Social. Portaria N69, de 14 de maio de 2020. Diário oficial da união (2020), Edição: 93, Seção 1, p.379. Available from: https://www.in.gov.br/web/dou/-/portaria-n-69-de-14-de-maio-de-2020-257197675

27. Ministério da Saúde, Secretaria de Atenção à saúde, Departamento de Atenção Básica. Manual sobre o cuidado à saúde junto a população em situação de rua. (2012), Série A. Normas e Manuais Técnicos, 98 p.: il.; Available from: http://189.28.128.100/dab/docs/publicacoes/geral/manual cuidado populalcao rua.pdf

28. Andrade JS, Vieira MJ. Prática assistencial de enfermagem: problemas, perspectivas e necessidade de sistematização. Rev Bras Enferm 2005;58(3):261-5. doi: 10.1590/S0034-71672005000300002 [Crossref]

29. Teixeira GA, Carvalho JBL, Silva ALMAD, Santos SBD, et al. Sistematização da assistência de enfermagem a pessoa em situação de rua. J Nursing UFPE 2015;9(3):7169-74. Available from: https://www.researchgate.net/deref/http\%3A\%2F\%2Fdx.doi.org\%2F10.5205\%2Freuol.7 505-65182-1-RV.0903201520

30. Brasil. Ministério da Saúde. Política Nacional de Atenção Básica. Brasília: Ministério da Saúde, 2012. Available from: http://189.28.128.100/dab/docs/publicacoes/geral/pnab.pdf

31. Priscilla MPM. Práticas coletivas de educação popular em saúde da familia na estratégia saúde da familia [Dissertação]. Belo Horizonte: UFMG; 2018. Available from: http://www.enf.ufmg.br/pos/defesas/1069M.PDF 
32. Secretaria de saúde, Governo do Estado do Ceará, nota técnica, recomendações para a população em situação de rua, (2020), [Internet]. Available from:

https://www.saude.ce.gov.br/wpcontent/uploads/sites/9/2018/06/nota tecnica recomen dacoes populacao em situacao de rua 2204 2020.pdf

33. Brasil. Ministério da Saúde. Recomendações para os consultórios na rua e a rede de serviços que atuam junto com a população em situação de rua, 2020. Available from: https://www.prefeitura.sp.gov.br/cidade/secretarias/upload/saude/saude mental covid 19.pdf

34. Brasil. Ministério da Saúde (MS). Secretaria de Atenção à Saúde. Política Nacional de Humanização da Saúde. Documento Base. $4^{\mathrm{a}}$ ed. Brasília: Ministério da Saúde (MS); 2007.

35. Gouveia EAH, Silva RO, Pessoa BHS. Competência cultural: uma resposta necessária para superar as barreiras de acesso à saúde para populações minorizadas. Rev Bras Educ Med 2019;43(1):82-90. doi: 10.1590/1981-5271v43suplemento1-20190066 [Crossref]

36. Silva FP, Silva EC, Ferreira AL, Frazão IDS. Segurança do paciente: ao alcance das pessoas em situação de rua? Rev Bras Enferm 2020; 73 (5): e20190114. doi: 10.1590/0034-7167-2019-0114 [Crossref]

37. Bogo PC, Bernardino E, Castilho V, Cruz EDA. O enfermeiro no gerenciamento de materiais em hospitais de ensino. Rev Esc Enferm USP 2015;49(4):632-9. DOI: doi: 10.1590/S0080-623420150000400014 [Crossref]

38. Cervieri NB, Uliana CH, Aratani N, Fiorin PM, Giacon BCC. O acesso aos serviços de saúde na perspectiva de pessoas em situação de rua. SMAD Rev Eletr Saúde Mental Álcool Drog [Internet]. 11 de novembro de 2019 [citado 2020 Nov 28];15(4):1-8.

Disponível em: http://www.revistas.usp.br/smad/article/view/164014

39. Lira CDG, Justino JMR, Paiva IKS, Miranda MGO, Saraiva AKM. O acesso da população em situação de rua é um direito negado? Rev Min Enferm 2019;23:e-1157. doi: 10.5935/1415-2762.20190004 [Crossref] 